\title{
FORMAÇÃo DE PSICÓLOGOS: ANÁLISE CURRICULAR ${ }^{(\mathrm{I})}$
}

\author{
Sandra Leal Calais ${ }^{1}$ \\ Elisabeth M. de Camargo Pacheco ${ }^{2}$
}

\section{Resumo}

\begin{abstract}
Objetivou-se caracterizar um curso de Psicologia de IES pública, São Paulo, analisando planos oficiais de ensino (1999) de suas disciplinas e estágios Análises curriculares revelaram que, de 4140 horas, $15,2 \%$ são de disciplinas de domínio conexo, 32,5\% básicas, 25,4\% específicas, 7,2\% pedagógicas e 19,6\% de estágios supervisionados. Houve predominância do enfoque uniteórico. Nas disciplinas básicas uniteóricas $44 \%$ tinham abordagem behaviorista, $19 \%$ psicossocial, $12,5 \%$ etológica, $12,5 \%$ biológica, $6 \%$ piagetiana e $6 \%$ teoria do ciclo vital; o ideal seria o enfoque multiteórico ou o equilíbrio. A maioria das disciplinas de domínio conexo tinha conteúdo relacionado à Psicologia, sugerindo preocupação do curso com a inter-relação. Nos estágios supervisionados, o predomínio do enfoque uniteórico mostra coerência na organização dos conteúdos de final de curso.
\end{abstract}

Palavras-chave: formação do psicólogo, análise curricular, psicologia.

\section{Psychologist's Graduation: an Analysis of the Curriculum}

\begin{abstract}
This research characterized a Psychology study course within a public university, by analyzing official course plans (1999), applicable to discipline and probation programs. The analysis revealed that in 4140 hours, $15.2 \%$ are connected domain disciplines, $32.5 \%$ basic disciplines, $25.4 \%$ specific disciplines, $7.2 \%$ pedagogical disciplines and $19.6 \%$ supervised probation programs. Within basic single-theory disciplines, over multi-theory disciplines, $44 \%$ adopted a behaviorist approach, $19 \%$ adopted a psychosocial approach, 12.5\% adopted an ethnological approach, 12.5\% adopted a biological approach, 6\% adopted a Piagetian approach and 6\% adopted the vital cycle theory; therefore the multi-theoretical approach would be the wiser choice. Almost all disciplines of the connected domain type had psychology-related contents, which suggests the studies concern with the interrelation of the various disciplines. As far as the probation programs are concerned, the predominance of uni-theoretical approach suggests coherence in the advanced years of the studies.
\end{abstract}

Key-words: psychologist formation, curriculum analysis, psychology.

\section{INTRODUÇÃ̃o}

\begin{abstract}
A Psicologia, como área de ensino e pesquisa, bem como profissão, é relativamente nova no Brasil. As Escolas Normais e o surgimento dos laboratórios experimentais e centros de desenvolvimento de testes e medidas psicológicas, são referências para o conhecimento histórico da Psicologia no Brasil, no final do século XIX e início deste século.

Segundo Buettner (1990, p. 24) "na década de 1930, com a criação da USP e de outras Universidades, inicia-se o período universitário da Psicologia brasileira".
\end{abstract}

Na década de 1940, acompanhando o crescimento universitário da Psicologia, surge um movimento de organização de profissionais, com a criação de instituições, associações de pesquisa e núcleos de estudo que, segundo van Kolck (1988), funcionaram como centros catalisadores da Psicologia.

Com a promulgação da Lei 4119, em 1962, iniciase o período profissional da Psicologia pois, por esse instrumento legal, foi regulamentada a profissão de Psicólogo ao mesmo tempo em que se definiram as

\footnotetext{
(I) As autoras agradecem a gentil contribuição da Profa. Dra. Geraldina Porto Witter.

${ }^{I}$ Docente da UNESP-Bauru - Bolsista CAPES-PICDT. Doutoranda em Psicologia como Profissão e Ciência-PUC-Campinas. E-mail: scalais@fc.unesp.br. Endereço: UNESP-Depto. de Psicologia - Faculdade de Ciências: Av. Engo. Luiz Edmundo Carrijo Coube, s/no. - CEP 17033-360 - Bauru - SP Fone/Fax: 14 - 2342888.

${ }^{2}$ Docente do Curso de Psicologia e Bolsista da PUC-Campinas. Doutoranda em Psicologia como Profissão e Ciência.
} 
disposições legais sobre a formação em Psicologia.

Nos últimos anos a proliferação de cursos superiores de Psicologia vem aumentando sem o correspondente aumento na qualidade.

Há um descompasso entre o profissional que temos e o que queremos formar. Branco (1998) chama a atenção para a condição do recém-formado: dispersão do saber, escassez de recursos materiais em trabalhos sociais, fantasia quanto à profissão liberal, grande competição entre os colegas e uma demanda que lhe exige ter clareza de seu papel.

Em que medida a Universidade brasileira tem contribuído para esclarecer esses aspectos é uma questão sobre a qual é necessário refletir.

A formação do Psicólogo tem sido alvo de inúmeros estudos e tema de discussão nacional e internacional, segundo Yukimitsu (1999).

Para Witter (1999), o ensino da Psicologia tem merecido o interesse de pesquisadores nacionais e estrangeiros, preocupados com questões relativas à formação e à atuação do Psicólogo. Tais estudos vêm consolidar uma discussão, que deve ser constante, referente à melhoria da qualidade de ensino nos cursos de formação de psicólogos, resultando em práticas profissionais críticas, atualizadas e atentas às demandas sociais

Yukimitsu (1999) salientou que a formação do psicólogo deve conter bases para pesquisas e práticas na área biológica, social e cognitiva-afetiva do comportamento: só se pode conceber a Psicologia em um corpo de conhecimentos científicos.

São inúmeros, segundo Duran (1994), os trabalhos que exemplificam a preocupação com a profissão de uma maneira geral, com as áreas de aplicação da Psicologia e com a necessidade de revisão da formação do profissional. Além dos trabalhos de autores como Witter et al (1992), Bastos (1993), Rocha (1993), Borges (1988), Buettner (1990) e outros, Duran ressalta que, também no âmbito das próprias instituições de ensino, podem ser verificados os movimentos de discussão curricular. Nos dias atuais, estes movimentos tornam-se quase que obrigatórios em todas as instituições de ensino de Psicologia, considerando-se a proposta do MEC quanto à implantação das novas diretrizes curriculares para o ensino superior. Ainda, para o mesmo autor, as mudanças curriculares, resultantes de um amplo processo reflexivo, são o caminho para viabilizarem-se transformações rápidas e de natureza tão variada.
Os cursos de Psicologia têm oferecido uma visão fragmentada porque grande parte dos seus currículos é construída segundo o interesse dos docentes, sem ter uma integração com um projeto maior de ensino institucional que os ampare.

Em que pesem os inúmeros e relevantes estudos sobre o tema, Barreto (1999, p. 15) considera que “... o Brasil está longe de contar com uma base de dados suficientemente ampla de resultados que possibilitem uma avaliação tão objetiva quanto possível do estado atual da Psicologia entre nós...". A mesma autora aponta a falta de trabalhos que considerem cada uma das múltiplas disciplinas ou domínios da Psicologia, além da tradicional divisão das áreas - clínica, escolar e organizacional - como, também, que considere a grande variedade de problemas referentes à formação acadêmica e ao exercício profissional.

Quanto ao tema específico deste trabalho, ao se proceder à análise de planos de ensino de um curso de Psicologia, não se deve perder de vista que, ao se analisarem conteúdos escritos sobre a atividade docente, não se quer dizer que não sejam transformados, em um processo dinâmico, dentro da sala de aula, pela atuação do professor e do aluno.

Muitas vezes as estruturas curriculares se revelam inadequadas e é preciso revê-las. Outras vezes a insatisfação com o "produto educacional" não deveria resultar na mera revisão curricular mas, também, no conhecimento amplo das condições contextuais que são oferecidas (ou não são) para a sua implementação.

Hoje é necessário que a formação do psicólogo seja geradora de um perfil que lhe possibilite ver o fenômeno psicológico na sua interdependência com o contexto sócio-cultural, atuar em equipes multidisciplinares, estar engajado nos movimentos de transformação social, gerando conhecimento e tecnologias apropriadas à realidade em que atua (Duran, 1994).

Resta saber se os cursos de formação de psicólogos refletem, em seu corpo curricular e em sua implementação, tais preocupações.

Para Gargantini (1996) a existência de um currículo é uma questão acadêmica e não somente legal e burocrática. Ensinar um conteúdo não é gerar cópias dele mas, sim, ensinar a fazer ciência, é recriá-lo.

Com o objetivo de estudar as variáveis relevantes na formação do psicólogo, o presente estudo foi reali- 
zado, sem a pretensão de esgotar o assunto, mas de oferecer uma pequena contribuição para as discussões, em especial no âmbito das Universidades, a respeito do profissional que está sendo formado.

Seu objetivo geral foi analisar os planos de ensino de um curso de Psicologia, de uma Instituição de Ensino Superior, com vistas a identificar suas variáveis relevantes, tal como se apresentavam no ano de 1999.

Os objetivos específicos foram:

a) identificar as categorias de disciplinas que compõem a grade curricular do curso de Psicologia da IES;

b) verificar o número de horas e de disciplinas, por semestre, de cada categoria identificada, comparando entre si os resultados obtidos;

c) conhecer o enfoque e a visão teórica das disciplinas que compõem a grade curricular do curso de Psicologia em estudo e

d) verificar a existência (ou não) de diferenças significativas entre os enfoques teóricos (uni e multiteórico) existentes dentro de cada categoria de disciplinas.

\section{MÉTODO}

No presente estudo foi analisado o curso de Psicologia da Faculdade de Ciências, de uma Instituição de Ensino Superior, de caráter público, localizada no interior do Estado de São Paulo.

Este curso, iniciado em 1969 como uma fundação e encampado pela IES em 1988, faz parte de um Câmpus que reúne três faculdades, com um total de 17 cursos e 3600 alunos.

A cada ano ingressam no curso de Psicologia 60 novos alunos, divididos em duas turmas: integral, com duração de cinco anos e o noturno, de seis anos. A estrutura curricular do curso permite a licenciatura ao final de quatro anos e a formação de psicólogo ao final de cinco/seis anos, dependendo do turno.

\section{Material}

Foram utilizados para esta análise dois tipos de documentos, cedidos pela seção técnica da IES: os planos de ensino oficiais das disciplinas do curso de licenciatura e os dos estágios supervisionados do curso de formação de psicólogos.

Estes planos se referiam a todas as disciplinas obrigatórias, optativas e estágios curriculares e estavam em vigor em 1999, com exceção das disciplinas pedagógicas alteradas na sua distribuição.

Dos planos de ensino constavam: identificação da disciplina, objetivos, conteúdo programático, metodologia básica, bibliografia básica, critérios de avaliação da aprendizagem, ementa e nome do professor responsável

\section{Procedimento}

A coleta de dados foi feita por meio da análise documental. O conjunto de disciplinas analisado foi dividido em quatro categorias de acordo com o seu conteúdo específico e sua denominação, sendo que os estágios supervisionados foram tratados em separado como é especificado nos itens seguintes:

- Disciplinas de Domínio Conexo: são as que oferecem informação e suporte à formação profissional em relação a outras áreas do saber e promovem enriquecimento curricular. Fazem a interface com a Psicologia. Segundo Oliveira (1992), alguns autores as denominam "disciplinas de formação básica” ou, como em Gonçalves (1994), "disciplinas de outras áreas de conhecimento”. Exemplos desta categoria são: Estatística, Sociologia, Antropologia, entre outras.

- Disciplinas Básicas: são as que provêem o repertório de conteúdo da Psicologia e do preparo científico e servem de apoio à psicologia como um todo, podendo ser, também, denominadas "disciplinas de formação fundamental" (Oliveira, 1992). Exemplos são: Psicologia Geral e Psicologia do Desenvolvimento.

- Disciplinas Específicas: são aquelas dirigidas à formação de competências específicas em Psicologia. Segundo Oliveira (1992), são as responsáveis pela formação profissional, podendo ser uniteóricas ou multiteóricas. Exemplos: Terapia Comportamental e Teorias e Técnicas Psicoterápicas.

- Disciplinas Pedagógicas: são as que complementam a formação para a licenciatura. Exemplos: Didática e Prática de Ensino.

- Estágios Supervisionados: são os que se caracterizam por práticas de intervenção, com supervisão docente, continuada e direcionada às diferentes áreas de atuação do psicólogo. Exemplos: Estágio em Psicologia Organizacional e Estágio em Psicologia Escolar. 
A partir dessa divisão, foram realizados três tipos de análise dos planos de ensino:

- número de horas e de disciplinas de cada categoria em cada um dos semestres do curso;

- verificação da existência ou não de relação de conteúdo das disciplinas de domínio conexo com a Psicologia e

- enfoque teórico das disciplinas básicas, específicas e o dos estágios supervisionados: uniteórico ou multiteórico; as de enfoque uniteórico foram avaliadas quanto a visão teórica empregada.

Após a análise, procedeu-se à entrevista com os professores responsáveis pelas disciplinas, com o intuito de verificar a adequação da avaliação dos planos de curso feita pelas pesquisadoras.

Foi excluída deste estudo a análise das disciplinas optativas, por razões de diferentes ordens: não identificação do professor responsável pela disciplina, não oferecimento regular da disciplina e aposentadoria do professor responsável.

A disciplina Educação Física, de natureza obrigatória (02 créditos), foi retirada da ordenação do conjunto de disciplinas mas constou no número de créditos.

\section{Resultados}

A partir dos dados coletados, evidenciaram-se os resultados que são descritos a seguir.

O curso-alvo deste estudo é desenvolvido em dez semestres, sendo os dois últimos de estágio supervisionado. O total de disciplinas é de 62 (61 disciplinas mais os estágios, considerados como uma disciplina).

Foi feito o levantamento, por semestre, do número de horas e de disciplinas e do total, referente a cada categoria de disciplinas estabelecida para este estudo, conforme pode ser visto na Tabela 1.

A Tabela 1 mostra que, do total de horas (4140), $15,2 \%$ são de disciplinas de domínio conexo, 32,5\% de disciplinas básicas, $25,4 \%$ de disciplinas específicas, 7,2\% de disciplinas pedagógicas e 19,6\% de estágios supervisionados. As disciplinas de domínio conexo somam 10 em todo o curso. As básicas são 26, as específicas são 20 e as pedagógicas quatro.

Nos três semestres iniciais há um maior número de horas de disciplinas de domínio conexo (600 horas), em relação às básicas (470 horas). A partir do $4^{\circ}$ semestre, um número maior de disciplinas e, por consequiência, de horas, refere-se às disciplinas específicas, seguidas pelas básicas e não mais são oferecidas as de domínio conexo. Somente no $6^{\circ}$ semestre têm início as disciplinas pedagógicas, que se estendem até o $8^{\circ}$ semestre.

Nos últimos dois semestres, que correspondem ao curso de Formação de Psicólogos, apenas duas disciplinas são oferecidas, além dos estágios supervisionados: uma disciplina básica e outra específica, com 30 horas cada uma.

O número de disciplinas por semestre varia de seis ( $4^{\circ}$ semestre) a oito (do $6^{\circ}$ ao $8^{\circ}$ semestres). As diferenças encontradas na distribuição das disciplinas em relação às quatro categorias estabelecidas foi significativa $\left(\mathrm{x}_{0}{ }_{0}=32.5, \mathrm{x}_{\mathrm{c}}{ }_{\mathrm{c}}=7,81, \mathrm{gl}=3\right.$ para $\left.\mu=0.05\right)$. Da mesma forma, foi significativa a diferença de distribuição da carga horária dentre as categorias e estágios supervisionados $\left(\mathrm{x}_{0}{ }_{0}=18.61, \mathrm{x}^{2}{ }_{\mathrm{c}}=9,49\right.$, $\mathrm{gl}=4$ para $\mu=0,05$ )

As disciplinas de domínio conexo são em número de onze: Filosofia, Antropologia, Sociologia, Biologia aplicada à Psicologia, Fisiologia Neuro-endócrina I e II, Metodologia Científica, Estatística Aplicada à Psicologia I e II, Saúde Pública e Saúde Mental, Ética Profissional.

A análise dos planos de ensino revelou que $90 \%$ dessas disciplinas têm relação de conteúdo com a Psicologia. Apenas uma disciplina (Biologia Aplicada à Psicologia), ainda que traga em sua denominação a referência de aplicabilidade à Psicologia, não está diretamente relacionada à ciência em questão, pelo menos pelo que consta no documento analisado.

O rol das disciplinas básicas e específicas foi avaliado quanto ao enfoque (uniteórico ou multiteórico) e quanto à visão teórica adotada.

As disciplinas básicas, em número de 25, bem como seus respectivos enfoques e visões teóricas predominantemente adotadas encontram-se no Quadro 1.

Os dados sintetizados no Quadro 1 revelam que $64,0 \%$ das disciplinas básicas têm um enfoque uniteórico e 36,0\% multiteórico e a diferença encontrada foi significante $\left(\mathrm{x}_{0}{ }_{0}=7.84, \mathrm{x}_{\mathrm{c}}=3,84, \mathrm{gl}=1\right.$ para $\left.\mu=0,05\right)$.

Ainda, quanto à visão teórica que fundamenta as disciplinas de enfoque uniteórico, 44,0 \% adotam uma linha behaviorista, $19,0 \%$ psicossocial, $12,5 \%$ etológica, $12,5 \%$ biológica, enquanto os enfoques piagetiano e o referente à teoria do ciclo vital, surgem com $6,0 \%$ cada.

O conjunto de disciplinas específicas totaliza 20. Seus enfoques e visões teóricas adotadas encontramse no Quadro 2. 
Tabela 1 - Distribuição do número de horas e de disciplinas por semestre e total

\begin{tabular}{|c|c|c|c|c|c|c|c|c|}
\hline \multicolumn{9}{|c|}{ Disciplinas } \\
\hline & \multicolumn{2}{|c|}{ Conexas } & \multicolumn{2}{|c|}{ Básicas } & \multicolumn{2}{|c|}{ Específicas } & \multicolumn{2}{|c|}{ Pedagógicas } \\
\hline Semestre & $\begin{array}{l}\text { Número } \\
\text { de discip. }\end{array}$ & $\begin{array}{c}\text { Horas / } \\
\text { Semestre }\end{array}$ & $\begin{array}{l}\text { Número } \\
\text { de discip. }\end{array}$ & $\begin{array}{c}\text { Horas / } \\
\text { Semestre }\end{array}$ & $\begin{array}{l}\text { Número } \\
\text { de discip. }\end{array}$ & $\begin{array}{c}\text { Horas/ } \\
\text { Semestre }\end{array}$ & $\begin{array}{l}\text { Número } \\
\text { de discip. }\end{array}$ & $\begin{array}{l}\text { Horas / } \\
\text { Semestre }\end{array}$ \\
\hline $1^{0}$ & 3 & 180 & 4 & 210 & & & & \\
\hline $2^{\circ}$ & 3 & 210 & 4 & 180 & & & & \\
\hline $3^{0}$ & 3 & 210 & 4 & 180 & & & & \\
\hline $4^{\circ}$ & & & 4 & 240 & 2 & 120 & & \\
\hline $5^{0}$ & & & 3 & 180 & 4 & 240 & & \\
\hline $6^{0}$ & 1 & 30 & 3 & 180 & 2 & 120 & 2 & 120 \\
\hline $7^{\circ}$ & & & 2 & 90 & 5 & 240 & 1 & 60 \\
\hline $8^{\circ}$ & & & 1 & 60 & 6 & 300 & 1 & 120 \\
\hline Sub-total & 10 & 630 & 25 & 1320 & 19 & 1020 & 4 & 300 \\
\hline Formação & & & 1 & 30 & 1 & 30 & & \\
\hline de Psicól. & & & & & & & & \\
\hline Total * & 10 & 630 & 26 & 1350 & 20 & 1050 & 4 & 300 \\
\hline
\end{tabular}

${ }^{*}$ Ao total geral de horas (3330) devem ser somadas horas de Estágio (810) e de Educação Física (30), perfazendo 4140 horas/curso total.

Os resultados apresentados no Quadro 2 mostram que $55,0 \%$ das disciplinas específicas, do primeiro ao oitavo semestres têm enfoque uniteórico e 45,0\% multiteórico. Esta diferença de enfoques não se caracterizou como significante $\left(\mathrm{x}_{0}{ }_{0}=1.0, \mathrm{x}_{\mathrm{c}}{ }^{2}=3,84, \mathrm{gl}=1\right.$ para $\mu=0,05)$. Das uniteóricas, $27,3 \%$ têm abordagem psicodinâmica e, com igual percentual aparecem as psicossociais e as sistêmicas. Com 9,0\% cada, aparecem as abordagens behaviorista e fenomenológica.

Em relação às disciplinas do estágio supervisionado (todas específicas), 75,9\% têm um enfoque uniteórico e $24,1 \%$ multiteórico. A diferença observada entre os enfoques teóricos foi significante $\left(\mathrm{x}^{2}=\right.$ $26.8, \mathrm{x}_{\mathrm{c}}{ }_{\mathrm{c}}=3.84, \mathrm{gl}=1$ para $\left.\mu=0.05\right)$. Entre as de enfoque uniteórico $38,1 \%$ têm abordagem psicodinâmica, $19 \%$ behaviorista, $28,5 \%$ psicossocial e $4,8 \%$ terapia centrada na pessoa, enquanto $9,6 \%$ abordagem sistêmica.

Das 60 disciplinas do curso, foram entrevistados docentes de 52 delas. Das restantes, os docentes estavam afastados por licença prêmio ( 2 disciplinas), aposentadoria (3), afastamento para chefia (1) ou sendo ministradas por um grupo de professores (2).
Dessas 52 disciplinas, somente houve discordância quanto à análise de enfoque e visão teórica em três delas: Psicopatologia I e II e Laboratório de Psicologia da Motivação, o que indica que foi viável detectar o enfoque das disciplinas pelos planos de ensino das mesmas.

\section{Discussão e CONClusões}

A análise documental pode ser valiosa quando se pretende complementar informações obtidas através de outros estudos e para revelar aspectos novos dos problemas. Outro aspecto importante, favorecedor desse tipo de análise, é a existência de algum vínculo entre o pesquisador e a instituição em que a pesquisa se desenvolveu (Buettner, 1990).

Os dados coletados no presente estudo revelaram aspectos interessantes de um curso de formação de psicólogos de uma Universidade pública de qualidade reconhecida.

Um dos aspectos verificados se refere às disciplinas pedagógicas que compõem o corpo curricular: elas 
Quadro 1 - Disciplinas básicas: enfoque e visão teórica

\begin{tabular}{|c|c|c|c|}
\hline \multirow[b]{2}{*}{ DISCIPLINAS } & \multicolumn{2}{|c|}{ ENFOQUE } & \multirow[b]{2}{*}{ VISÃOTEÓRICA } \\
\hline & Multiteórico & Uniteórico & \\
\hline História dos Sistemas & $x$ & & - \\
\hline Psicologia Geral & $x$ & & - \\
\hline Psicologia Experimental I & & $x$ & Behaviorismo \\
\hline Etologia & & $x$ & Etologia \\
\hline Psicologia do Desenvolv. I & & $x$ & Piaget \\
\hline Laboratório Psico. Exper. II & & $x$ & Behaviorismo \\
\hline Psicologia do Desenvolv. II & $x$ & & - \\
\hline Psicologia Experimental II & & $x$ & Behaviorismo \\
\hline Laboratório Psico. Exper. II & & $x$ & Behaviorismo \\
\hline Psicologia do Desenvolv. III & & $x$ & Teoria do Ciclo Vital \\
\hline Psicologia da Motivação & & $x$ & Behaviorismo \\
\hline Laboratório Psico. da Motiv. & $\mathrm{X}$ & & - \\
\hline Técnicas de Observação & & $x$ & Behaviorismo \\
\hline Psico.da Percepção e Memória & & $x$ & Behaviorismo \\
\hline Labor. de Psico. Percep. e Mem. & $\mathrm{X}$ & & - \\
\hline Psicologia da Personalidade I & $x$ & & - \\
\hline Psicologia Social I & $x$ & & - \\
\hline Psicologia da Personalidade II & & $x$ & Sócio-histórica \\
\hline Psicologia Social II & & $x$ & Sócio-histórica \\
\hline Psicopatologia I & & $x$ & Biologia \\
\hline Psicopatologia II & & $x$ & Biologia \\
\hline Psicologia e Comunidade & & $x$ & Sócio-histórica \\
\hline Desenvol. e Educação Sexual & & $x$ & Etologia \\
\hline Questões da Sexualidade Hum. & $x$ & & - \\
\hline Dinâmica de Grupo & $\mathrm{X}$ & & - \\
\hline
\end{tabular}

são oferecidas estritamente de acordo com o que a legislação determina, tanto no que se refere ao número de disciplinas presentes na grade curricular, quanto à carga horária de cada uma dessas disciplinas. Isso denota a importância apenas formal dada à licenciatura nesse curso.

O conjunto de disciplinas e carga horária estão direcionados à formação do profissional. Revelam, na sua organização, a preocupação de manter conteúdos que, de fato, auxiliem nessa formação. Isto pode ser verificado na relação de conteúdo existente entre as disciplinas de domínio conexo e a Psicologia como, também, na divisão equilibrada dos enfoques teóricos entre as disciplinas específicas.

Ressalte-se que o conjunto das disciplinas básicas tem como característica significativa a uniteoricidade. Este aspecto mostra-se contraditório em relação à característica que deve ter o conjunto de disciplinas: ele deve oferecer ao aluno uma visão ampla de grandes temas e teorias em Psicologia e não uma visão baseada em uma ou outra teoria. Isto poderá conduzir o aluno, prematuramente, à adoção de preferências por um ou outro modelo teórico, sem conhecer as alternativas existentes. Evidentemente que só em casos específi- 
Quadro 2- Disciplinas específicas: enfoque e visão linha

\begin{tabular}{|c|c|c|c|}
\hline \multirow[b]{2}{*}{ DISCIPLINAS } & \multicolumn{2}{|c|}{ ENFOQUE } & \multirow[b]{2}{*}{ LINHA } \\
\hline & Multiteórico & Uniteórico & \\
\hline Técn. de Exame Psicológico II & $x$ & & \\
\hline Psicomotricidade I & $x$ & & \\
\hline Psicomotricidade II & $x$ & & \\
\hline Psicologia do Excepcional I & $x$ & & \\
\hline Psicologia do Excepcional II & & $x$ & Behaviorismo \\
\hline Psicologia Escolar I & $x$ & & \\
\hline Psicologia Escolar II & & $x$ & Sócio-histórica \\
\hline Psicologia das Rel. Humanas & & $x$ & Sistêmica \\
\hline Orientação Vocacional I & $x$ & & \\
\hline Orientação Vocacional II & & $\mathrm{X}$ & Sócio-histórica \\
\hline Teorias e Técn. Psicoterápicas I & & $x$ & Psicodinâmica \\
\hline TTP II & & $\mathrm{X}$ & Psicodinâmica \\
\hline TTP III- Terapia Comportam. & & $x$ & Behaviorismo \\
\hline TTP IV- Terapia Comportam. & & $x$ & Behaviorismo \\
\hline TTP V- Fenomenologia & & $x$ & Fenomenologia \\
\hline TTP VI- Psicoterapia & $x$ & & \\
\hline TTP VII- Psicanálise & $\mathrm{X}$ & & Psicodinâmica \\
\hline TTP VIII- Terapia Familiar & & $x$ & Sistêmica \\
\hline Psicologia Organizacional & & $x$ & Sistêmica \\
\hline
\end{tabular}

cos é de se esperar a unicidade. Ex. Terapia Comportamental - behaviorismo.

A ocorrência equilibrada de enfoques uniteóricos e multiteóricos no conjunto de disciplinas específicas, denota uma importante correção de rumos na formação do aluno em relação ao que ocorre com as disciplinas básicas. Aquelas disciplinas são oferecidas a partir do $4^{\circ}$ semestre do curso, num momento em que, em tese, esperam-se alunos com mais maturidade para os estudos, com maior domínio da nova realidade educacional e dos elementos formais e informais do próprio curso.

O predomínio das disciplinas básicas sobre as demais permite inferir que há uma valorização das temáticas que visam a formação geral do psicólogo, como premissa da organização do corpo curricular. Embora isto seja desejável, sugere-se a revisão do enfoque teórico predominante - de uniteórico para multiteórico - pois grandes temas e teorias em Psico- logia, igualmente importantes, podem ser encontrados, na literatura, com diferentes abordagens.

Ao se verificar o conjunto referente aos estágios supervisionados, ele se mostra coerente quanto ao enfoque teórico utilizado: predominantemente uniteórico. Neste momento do curso, a formação do aluno já está dirigida a áreas específicas, com diferenciação de linha teórica havendo, simultaneamente, a prática de intervenção, mesmo que supervisionada.

Tal como aponta Barreto (1999) em sua pesquisa sobre formação e exercício profissional dos psicólogos (CRP $-6^{\text {a }}$ região), os sujeitos por ela pesquisados sugeriram que os estágios supervisionados deveriam ter “...maior carga horária, possibilitando uma maior atuação, além de uma diversificação maior das áreas propostas" (p. 50).

No curso ora estudado, os estágios supervisionados aparecem em terceiro lugar, do ponto de vista do total de horas a eles destinado. Justifica-se tal carga horária pelo fato dos estágios serem 
oferecidos, como nos demais cursos, apenas nos dois últimos semestres.

Com a possibilidade presente de revisão curricular, em função das novas diretrizes a serem definidas, pergunta-se se não seria plausível pensar na iniciação do aluno (em diferenciados níveis de prática) em situações que se assemelhassem aos estágios da fase terminal do curso. Obviamente deveriam ser consideradas as competências pessoais e acadêmicas dos alunos, em cada semestre do curso.

Ao ser analisado o referencial teórico de cada disciplina, não se tem acesso ao modo como foi trabalhado, na relação com o aluno, o conteúdo especificado nos planos de ensino.

Outro ponto a ser considerado é que nem tudo o que está previsto no plano de ensino, se operacionaliza em sala de aula devido às dificuldades institucionais,

\section{REFERÊNCIAS}

Barreto, M. F. M. (1999). A Formação, o Exercício Profissional do Psicólogo e a Prática da Dinâmica de Grupo (Tese de Doutorado). Pontifícia Universidade Católica de Campinas.

Branco, M.T.C.,(1998). Que Profissional Queremos Formar? - Psicologia Ciência e Profissão,18(3), 28-35

Buettner, G. E. B. P. von (1990). Análise da Estrutura Curricular de um Curso de Psicologia: Subsídios para Reestruturação (Dissertação de Mestrado). Universidade Estadual de Campinas.

Duran, A. P. (1994). Alguns Dilemas na Formação do Psicólogo: Buscando Sugestões. In: Conselho Federal de Psicologia, Psicólogo Brasileiro: práticas emergentes e desafios para a formação. São Paulo: Casa do Psicólogo.

Gargantini, M. B. M. (1996). Formação do Fonoaudiólogo: Análise Curricular (Dissertação de Mestrado). Pontifícia Universidade Católica de Campinas.

Gonçalves, C. L. C. (1999). Formação Geral e Estágio Acadêmico em Psicologia Escolar. In: C. Witter (Org.) Ensino de Psicologia. Campinas, SP: Alínea.

Gonçalves, C. L. C. (1994). Formação e Estágio Acadêmi- às peculiaridades do corpo discente e ao efetivo envolvimento do professor com a disciplina e com os alunos. Não foi objeto deste estudo identificar tais dificuldades, o que não impede a realização de novas pesquisas que abordem essas questões.

Entende-se que a colaboração oferecida por esta pesquisa poderá ser um referencial para o início das discussões, dentro do próprio curso, com a participação de todos os envolvidos no processo de formação do futuro profissional de Psicologia.

No momento atual, com as novas diretrizes curriculares que deverão ser implantadas em breve, conhecer a estrutura do curso que se oferece e utilizar tal conhecimento para prepará-lo para os cenários futuros, é tarefa de grande importância e, principalmente, caracteriza-se como uma atitude de compromisso institucional com a formação do psicólogo.

co em Psicologia Escolar no Brasil: Análise curricular (Dissertação de Mestrado). Pontifícia Universidade Católica de Campinas, Campinas.

Oliveira, F. A. F. (1992). Formação do Psicólogo Escolar em duas Instituições de Ensino Superior: Análise através dos planos de disciplinas (Dissertação de Mestrado). Pontifícia Universidade Católica de Campinas.

Silva, C. M. (1994). Estatística Aplicada à Psicologia e Ciências Sociais. Portugal: Mc Graw-Hill

Van Kolck, O. L. (1988). Contribuição à História da Psicologia em São Paulo. Revista Brasileira de Pesquisa em Psicologia, 1(1), 7-11.

Witter, C. (Org.) (1999). Ensino de Psicologia Campinas, SP: Alínea.

Yukimitsu, M. T. C. P. (1999). A Formação do Psicólogo: Considerações Gerais. In: C.Witter (Org.) Ensino de Psicologia. Campinas, SP: Alínea.

Recebido em: 31/10/2000

Revisado em: 29/11/2001

Aprovado em: 14/03/2001 